\section{Pneumokokken: Einsatz hoch valenter Konjugatimpfstoffe}

Savulescu C et al. Effect of high-valency pneumococcal conjugate vaccines on invasive pneumococcal disease in children in SpIDnet countries: an observational multicentre study. Lancet Respir Med 2017; 5: 648 - 648

Seit der Einführung hoch-valenter Pneumokokken-Impfstoffe ist die Inzidenz invasiver Erkrankungen durch Pneumokokken bei Kindern deutlich gesunken. Gleichzeitig nimmt jedoch die Zahl der Erkrankungen durch Erreger, die nicht von diesen Impfstoffen abgedeckt werden, zu, so das $\mathrm{Er}$ gebnis der Studie von Camelia Savulescu und Kollegen des Streptococcus pneumoniae Invasive Disease network (SpIDnet).

Die ersten Pneumokokken-Konjugatimpfstoffe (PCV) enthielten Antigene von 7 Serotypen (PCV7). Im Jahr 2009 wurden 2 weitere Konjugatimpfstoffe in Europa zugelassen: ein 10-valenter PCV (PCV10) sowie ein 13-valenter (PCV13).

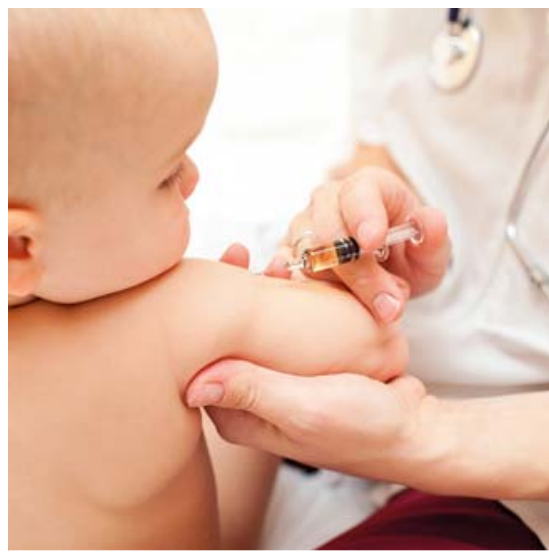

Durch die hoch-valente Konjugatimpfstoffe sank die Anzahl an PneumokokkenErkankungen bei Kleinkindern. Die SpIDnet-Daten zeigen aber auch, dass die Zahl der Erkrankungen durch Erreger, die durch die Impfstoffe nicht abgedeckt werden, zunimmt.
Mit ihnen kann ein größeres Spektrum von Serotypen abgedeckt werden. SpIDnet beobachtet und untersucht die Inzidenz invasiver Erkrankungen durch Pneumokokken an 9 Standorten in Europa. An 5 dieser Standorte wird für die Impfung PCV13 verwendet; die 4 restlichen Standorte nutzen den PCV10 in Kombination mit PCV13. An sechs der Standorte wurden ca. 90\% der Kinder geimpft, bei den restlichen 3 konnte eine Impfquote von 67-78\% erreicht werden.

In ihrer Studie verglichen die Forscher die Inzidenz invasiver Erkrankungen durch Pneumokokken in den 4 Jahren nach der Einführung von PCV13 sowie PCV10 in Kombination mit PCV13 mit der durchschnittlichen Inzidenz in den vorangegangenen Jahren, in denen PCV7 zur Impfung benutzt worden war. Hierfür errechneten sie die Inzidenzrate (Incidence Rate Ratio [IRR]) sowie die 95\%-Konfidenzintervalle (KI) für jedes Jahr und führten eine Random-EffectsMetaanalyse mit den gepoolten Werten aller Standorte durch.

\section{Ergebnisse}

4 Jahre nach der Einführung von PCV13 und PCV10 in Kombination mit PCV13 lag die gepoolte IRR für eine invasive Pneumokokken-Erkrankung bei Kindern unter 5 Jahren bei 0,53 (95\%-KI=0,43$0,65)$ für alle Serotypen. Für Erkrankungen, die durch PCV7-Serotypen hervorgerufen werden, betrug die IRR 0,16 (95\%-KI=0,07-0,40); für Erkrankungen durch die Serotypen 1, 5 oder 7F lag die IRR bei 0,17 (0,07-0,42); für Erkrankungen durch die Serotypen 3, 6A und 19A betrug die IRR 0,41 (95\%-KI=0,25-0,69). Die Ergebnisse waren ähnlich an den Standorten, an denen nur PCV13 verwendet wurde. Bei Erkrankungen durch Serotypen, die nicht von PCV13 abgedeckt werden, kam es zu einem Anstieg um $62 \%(I R R=1,62 ; 95 \%-K I=1,09-2,42)$.
FAZIT

Während die Inzidenz invasiver Erkrankungen durch Pneumokokken seit der Einführung hoch valenter Konjugatimpfstoffe allgemein abnimmt, konnte diese Studie zeigen, dass Erkrankungen durch Serotypen, die nicht vom Impfstoff abgedeckt werden, häufiger werden. Dies ist vermutlich durch Serotypen-Replacement bedingt, so die Interpretation der Autoren. Sie empfehlen daher, die weiteren Auswirkungen der PCV10und PCV13-Impfprogramme auf lange Sicht sorgsam zu überwachen.

Kathrin Strobel, Mannheim 